\title{
CONSTRUÇÃO E USO DO PULVERIZADOR-LOGARÍTMICO MANUAL
}

\author{
F. S. DE ALMEIDA $\left({ }^{1}\right)$, B. N. RODRIGUES $\left({ }^{1}\right)$, A. CATANEO(2) \& V. F. DE OLIVEIRA(1)
}

(1) Pesquisadores da Fundação Instituto Agro nô mico do Paraná - IAPAR. Londrina, PR, da Area de Herbologia e Botânica.

(2) Analista de Sistema da UNESP - Botucatu, SP.

\section{RESUMO}

Descreve-se a construção do pulverizador-logarítmico, seu funcionamento e gama de aplicações. Deduzem-se as fórmulas matemáticas em que o mesmo se baseia. Apresentam-se ábacos que permitem determinar a concentração de calda em qualquer ponto da área pulverizada.

UNITERMOS: controle de ervas, herbicidas, equipamentos, pulverizador logarítmico.

\section{SUMMARY}

\section{CONSTRUCTION AND USE OF THE MANUAL} LOGARITHMIC SPRAYER

The construction of a logaritmic-sprayer, its functioning, and range of applications are described. The mathematic formulas in which it is based are inferred. The abacus that allows the solution concentration determination in any point of the treated area is presented.

KEYWORDS: weed control, herbicides, equipment, logarithmic sprayer.

\section{INTRODUÇÃO}

O pulverizador-logarítmico foi concebido para ser utilizado em estudos preliminares de novos produtos fitossanitários, de forma a determinar as doses de controle dos agentes a que se destinam, e as de seletividade para as culturas. O aparelho pode também servir para teste de mistura de dois produtos quando se mantém fixa a dose de aplicação de um deles.

Os primeiros pulverizadores-logaritmicos que apareceram no mercado eram aparelhos complexos, dispondo de locomoção motorizada, ou montados em veículos, o que lhes assegurava uma deslocação e velocidade uniforme, mas exigia grandes áreas de aplicação.

Mais tarde, Ries e Terry (4), simpli- ficaram a construção e apresentaram um modelo manual que tinha a vantagem de trabalhar em condições de irregularidade de terreno, onde os outros não tinham acesso, além de poder utilizar parcelas de pequenas dimensões. Posteriormente, Cialone e Bayer (1) introduziram-lhe algumas modificações, melhorando a precisão do aparelho. A construção do pulverizador descrita por Cialone et al. (2), e Cialone e Davis (3) apresentam algu mas sugestões do seu uso e limitações.

Nenhum deles, porém, descreve as bases matemáticas que regulam o funcionamento do aparelho, o que se pretende relatar no presente trabalho.

\section{DESCRIÇÃO DO PULVERIZADOR-LOGARÍT MICO}

O pulverizador-logarítmico baseia-se no princípio da diluição contínua e uniforme de um produto, de forma a que a concentração se reduza ao longo do tempo, segundo uma função conhecida. Assim, desde que a velocidade de deslocação do aparelho seja constante, o tempo de aplicação relaciona-se com a distância percorrida, o que permite determinar, mais tarde, a dose aplicada em qualquer ponto do percurso, a partir da distância que o separa do local de partida.

$\mathrm{Na}$ Figura 1 apresenta-se o esquema do aparelho. Consta, essencialmente, de uma fonte de pressão, ar comprimido ou $\mathrm{CO}_{2}$ (1), e dois reservatórios, usualmen te frascos de vidro, sendo um maior (6) destinado ao diluente e outro (7) ao produto que se vai testar e do qual se conhece a concentração. Os dois frascos encon- 
tram-se, normalmente, dentro de uma caixa, o que facilita o seu transporte, mas cuja finalidade principal é a de proteger o operador dos estilhaços dos recipientes, caso estes se quebrem devido à pressão a que estão submetidos. Os reservatórios são providos de tampas atravessadas por dois tubos, sendo que um atinge o fundo do recipiente e outro fica ao nivel do líquido que o enche. No reservatório de diluente, o mais curto é o da entrada do gás comprimido e o outro, o da saída do liquido. Este encontra-se provido de registro de passagem (5), que permite que a ligação com o recipiente de concentrado só se faça quando se inicia a pulverização. No reservatório de concentrado o tubo mais comprido dá entrada ao diluente e, pelo mais curto, sai a calda diluída. Este liga-se por mangueira à lança de pulverização (8) equipada com bicos pulverizadores em número e tipo que melhor se adapte às dimensões da parcela e especificações de pulverização.

A ligação entre os dois reservatórios faz-se através de mangueira, assim como entre o recipiente de diluente e o botijão de gás comprimido. Este encontra-se equipado com manômetro (2) e válvula reguladora de pressão (4).

A concepção do aparelho é bastante simples e na sua construção utiliza-se material e peças usualmente usadas nos pulverizadores comuns. As únicas que, necessariamente, têm que ser construídas são as tampas dos reservatórios, que podem ser fabricadas em qualquer serralheria.

A exigência de deslocação do aparelho a velocidade constante, obtêm-se treinando o operador a manter o passo uniforme. Um bom auxiliar para tal é o uso de metrônomo, do tipo adotado nas escolas de música o qual, colocado perto do local de pulverização, permite que o operador regule o passo pelo som cadenciado do aparelho.

\section{DESENVOLVIMENTO TEÓRICO}

Usando como concentrado uma solução de concentração conhecida, normal-

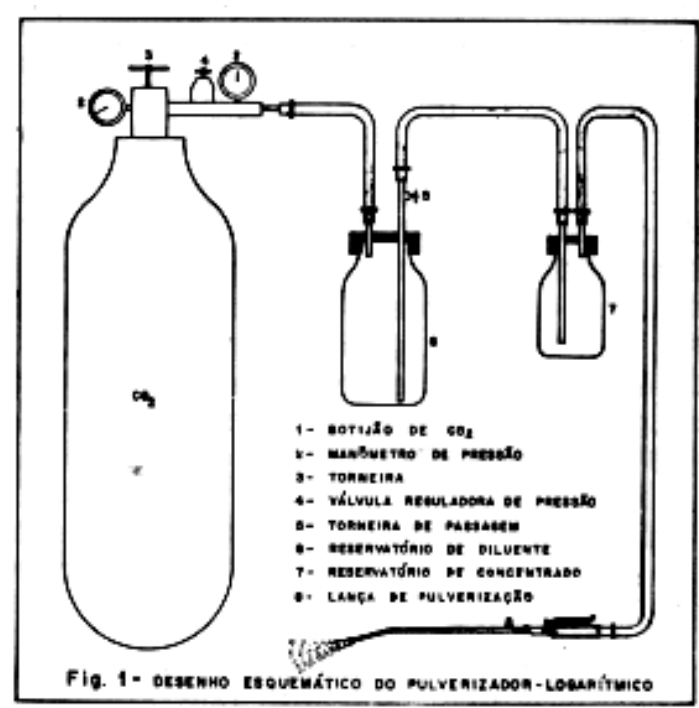

mente o $\mathrm{Ca} \mathrm{C} 12$, e como diluente água destilada, pode calcular-se a concentração da calda ao longo da distância percorrida pelo pulverizador, recolhendo o líquido pulverizado em placas de Petri, colocadas a intervalos regulares, determinando, depois, a concentração em cada uma delas, com refratômetro.

Relacionando essas concentrações com as distâncias, obtêm-se uma função do tipo exponencial, que se pode expres sar pela equação:

$$
y=a e^{b x}
$$

em que :

$\mathrm{y}=$ concentração à distância $\mathrm{x}$

$\mathrm{a}=$ concentração inicial

$\mathrm{e}=$ número neperiano

$\mathrm{b}=$ taxa de variação da concentração

$\mathrm{x}$ - distância percorrida

A taxa de variação da concentração b expressa-se por uma fração. Assim se for $1 / 20=0,05$, significa que, por unidade de distância, a variação da concentração é de $5 \%$.

No caso do pulverizador-logarítmico, a variação da concentração é provocada pela entrada, a vazão constante, do diluente no recipiente do concentrado. Designando por $\mathrm{v}$ a vazão do diluente e por $\mathrm{V}_{\mathrm{c}} \mathrm{o}$ volume do concentrado, a taxa de 
variação da concentração é, pois, v/vc. $\mathrm{O}$ expoente bx toma, então, a forma:

$$
\mathrm{bx}=\frac{\mathrm{v}}{\mathrm{v}_{\mathrm{c}}} \mathrm{x}
$$

Mas v.x., isto é, o produto da vazão do diluente pela distância percorrida pelo aparelho, corresponde à quantidade de diluente gasto desde que se inicia a operação até se atingir o ponto localizado à distância $\mathrm{x}$, e que se designa por vd. O expoente da função exponencial pode, por isso, expressar-se por :

$$
\mathrm{bx}=\frac{v_{\mathrm{d}}}{v_{\mathrm{c}}}
$$

Dado que a variação da concentração é decrescente o expoente tem sempre o sinal negativo.

Para facilidade de exposição usa-se os símbolos $\mathrm{Cd}$ para representar a concentração da calda à distância d e CI para a concentração inicial, pelo que, a função exponencial toma a forma:

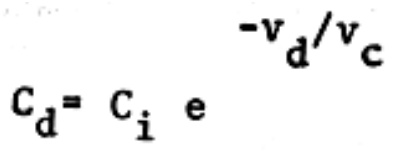

Aplicando logarítmico a esta função tem-se :

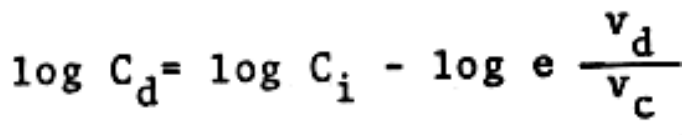

como $\log \mathrm{e}-0,4343$,

$$
\log C_{d}=\log C_{i}-0,4343 \frac{v_{d}}{v c}
$$

Esta expressão permite determinar a concentração da calda em qualquer ponto da área pulverizada, uma vez que se conhece a concentração inicial do concentrado $(\mathrm{CO}$ e o volume de diluente gasto até esse ponto (vd ), e o de concentrado $\left(\mathrm{V}_{\mathrm{C}}\right)$, determinam-se da forma que a seguir se descreve.

\section{CALCULO DO VOLUME DE DILUENTE}

Pela própria construção do aparelho
(Figura 1) verifica-se que, quando do final da pulverização, o volume de líquido no reservatório de concentrado mantémse inalterado. Portanto, a quantidade de calda utilizada para pulverizar uma parcela é igual ao volume de diluente gasto na operação.

Sendo A a área da parcela em $\mathrm{m}^{2}$ e D a vazão do pulverizador em //ha, o volume de diluente vd que se gasta para pulverizar essa área é dado pela expres são:

$$
v_{d}^{*}=\frac{\text { D.A }}{10000} 1 \text { itros }
$$

Como normalmente as parcelas experimentais são retangulares, a área é igual ao produto da largura 1 (m) pelo comprimento d (m), pelo que :

$$
v_{d}=\frac{D \cdot l \cdot d}{10000} 1 \text { itros (2) }
$$

Uma vez escolhidas as dimensões da parcela e a vazão com que se pretende trabalhar, esta fórmula permite determinar o volume de diluente de que se necessita dispor.

\section{CALCULO DO VOLUME DE CONCENTRADO}

Para calcular o volume de concentrado é preciso definir-se, previamente, a concentração que se pretende que a calda atinja no final da pulverização. Admitase que seja de $1 / z$ da concentração inicial. $\mathrm{Na}$ fórmula 1, Cd toma, então, o valor de $\mathrm{C} \mathrm{j} \mathrm{/} z$, obtendo-se :

$$
\log \frac{\mathrm{C}_{i}}{z}=\log \mathrm{C}_{i}-0,4343 \frac{\mathrm{v}_{\mathrm{d}}}{v_{c}}
$$

ou, tirando o valor de $\mathrm{v}_{\mathrm{C}}$ :

$$
v_{c}=\frac{0,4343}{\log z} v_{d}
$$

Uma vez que vd já é conhecido, esta expressão permite determinar o volume de concentrado a utilizar para atingir-se uma dada concentração final.

Definiu-se a concentração final como 
$\mathrm{Cd}=\mathrm{Cj} / \mathrm{z}$, ou o que é o mesmo $\mathrm{Cd} / \mathrm{C}_{\mathrm{i}}=$ $1 / z$. Fazendo na fórmula $3, z=2,4 \ldots 2 n$, obtêm-se os valores de $\mathrm{v}_{\mathrm{C}}$ para as concentrações finais $\mathrm{Cd} / \mathrm{C}_{\boldsymbol{i}}=1 / 2 \mathrm{n}$ :

$$
v_{c}=\frac{0,4343}{\log 2^{n}} v_{d}
$$

ou, tirando o valor de vd/vc :

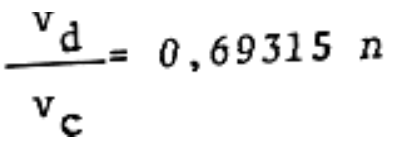

No Quadro 1 encontram-se tabelados os valores que vd/vc toma para as diferentes concentrações finais $\mathrm{Cd} / \mathrm{C}_{\boldsymbol{i}}$, e também as meias-doses (MD) correspondentes, cuja definição será dada na seção "construção de ábacos".

Utilizando esse quadro pode calcularse diretamente o valor de $\mathrm{V}_{\mathrm{C}}$. Assim, para a concentração final de $1 / 16$, o quadro indica que vd/vc $-2,773$. Segundo vd conhecido, o volume de concentrado a utilizar é vc - vd/2,773.

Quadro 1. Relação das Meias-Doses (MD) e Concentração Final (Cd/Ci) com os Volumes de Diluentes e Concentrado $(\mathrm{vd} / \mathrm{vc}) \cdot$

\begin{tabular}{rcc}
\hline $\mathrm{MD}$ & $\mathrm{Cd} / \mathrm{Ci}$ & $\mathrm{vd} / \mathrm{vc}$ \\
\hline $\mathrm{MD}$ & $1 / 2$ & 0,693 \\
2MD & $1 / 4$ & 1,386 \\
3MD & $1 / 8$ & 2,079 \\
4MD & $1 / 16$ & 2,773 \\
5MD & $1 / 32$ & 3,466 \\
6MD & $1 / 64$ & 4,159 \\
$7 \mathrm{MD}$ & $1 / 128$ & 4,852 \\
8MD & $1 / 256$ & 5,545 \\
$\mathrm{nMD}$ & $1 / 2 \mathrm{n}$ & $0,693 \mathrm{n}$ \\
\hline \hline
\end{tabular}

CAPACIDADE DOS RESERVATÓRIOS DO PULVERIZADOR-LOGARÍTMICO

A capacidade dos reservatórios de diluente e de concentrado pode ser igual ou superior à dos volumes calculados pela forma indicada anteriormente.

A primeira hipótese é a mais comum pois, não sendo igual, obriga a medir o diluente em cada operação, o que se torna fastidioso. Exige, porém, que se disponha de um reservatório de concentra- do para cada concentração final com que se pretende trabalhar. Como as mais usadas são as de $1 / 16$ e $1 / 64$, os pulverizadores-logarítmicos estão, normalmente, equipados com dois reservatórios de concentrado, com capacidade correspondente a essas diluições.

Também há conveniência em usar no reservatório de diluente o volume exato que se vai gastar na pulverização da parcela, pois permite controlar a velocidade de deslocação do operador. Se for correta, a sua chegada final da parcela coincide com o esgotamento do diluente, começando a sair gás pelos bicos do pulverizador.

\section{CONSTRUÇÃO DE ÁBACOS}

Uma vez conhecidos os volumes de diluente e concentrado, a fórmula 1 permite determinar a concentração a que a calda é aplicada em qualquer ponto da parcela. Quando, porém, se trabalha com um número elevado de tratamentos, es ses cálculos tornam-se trabalhosos, pelo que se usam ábacos, em que a curva logaritmica se encontra desenhada, usando as distâncias como abcissa e as doses como ordenada.

A curva define-se calculando pontos a partir da fórmula 1 ou, mais expeditamente, determinando a "distância da meia-dose".

Entende-se por distância da meia-dose, e designa-se por d(MD ), a distância a que a concentração inicial se reduz a metade. Ela é calculada substituindo vd da fórmula 3, pelo valor dado pela função 2, obtendo-se :

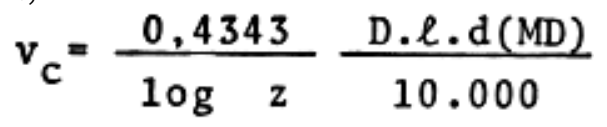

Fazendo $z=2$ e tirando o valor de d(MD) :

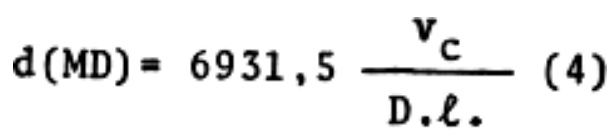

Para se calcular a distância a que a concentração se reduz a $1 / 4,1 / 8, \ldots$, 1 /2n da inicial, a que se dá o nome de "distância de 2 meias-doses, d(2MD) ", 
"distância dg 3 meias-doses, d(3MD) ", ... "distância de $\mathrm{n}$ meias-doses, d(nMD) " ( Quadro 1), atribui-se a $z$ os valores 4, $\mathbf{8}, \ldots, 2 \mathrm{n}$ :

$$
\begin{aligned}
& v_{c}=\frac{0,4343}{\log 2^{n}} \frac{D \cdot \ell \cdot d(n M D)}{10 \cdot 000} \\
& d(n M D)=n 6931,5 \frac{v_{c}}{D \cdot \ell}
\end{aligned}
$$

Aplicando-se a fórmula 4, obtêm-se :

$$
\mathrm{d}(\mathrm{nMD})=\mathrm{nd}(\mathrm{MD})
$$

Esta expressão indica que a distância da dose nMD é igual a $\mathrm{n}$ distâncias da meia-dose, donde se pode concluir que a cada distância percorrida equivalente a meia-dose, a concentração da calda reduz-se a $2,3, \ldots, n$ meias-doses, ou seja, a $1 / 4,1 / 8, \ldots, 1 / 2 \mathrm{n}$ da inicial.

No Quadro 1 encontra-se a relação entre meias-doses e as concentrações correspondentes.

Por outro lado, analis ando a fórmula 4 , verifica-se que, sendo constante a vazão do aparelho e a largura da parcela, a distância da meia-dose é função, apenas, do volume de concentrado, o qual, como já foi demonstrado, determina a concentração final.

Estas duas características do pulverizador-logarítmico permitem a construção expedita de ábacos para cada concentração final com que se pretende trabalhar.

As mais usuais, como foi dito, são as de $1 / 8$, e $1 / 64$.

Para a construção do ábaco de concentração final igual a de $1 / 16$ da inicial, marca-se no eixo das abcissas, usando qualquer escala, a distância das 4 meiasdoses, d(4MD ). Divide-se essa distância em 4 partes iguais, correspondentes à d(MD ), d(2MD ), e d(3MD) . No eixo das ordenadas, marca-se a concentração inicial, também em qualquer escala, dividindo-a em 1/2, 1/4, 1/8 e 1/16, correspondentes, respectivamente, à $\mathrm{MD}, 2 \mathrm{MD}$,
$3 \mathrm{MD}$, e 4MD, tal -como está representado na Figura 2.

$\mathrm{Na}$ construção do ábaco para concentração final de $1 / 64$, procede-se de maneira idêntica, só que a abcissa é dividida em 6 partes iguais e, na das ordenadas, marcam-se as doses até 1/64 (Figura 3).

Para sua utilização basta, então, atribuir a MD, 2MD, ... e, e a d(MD), $\mathrm{d}(2 \mathrm{MD}), \ldots$, os seus valores reais.

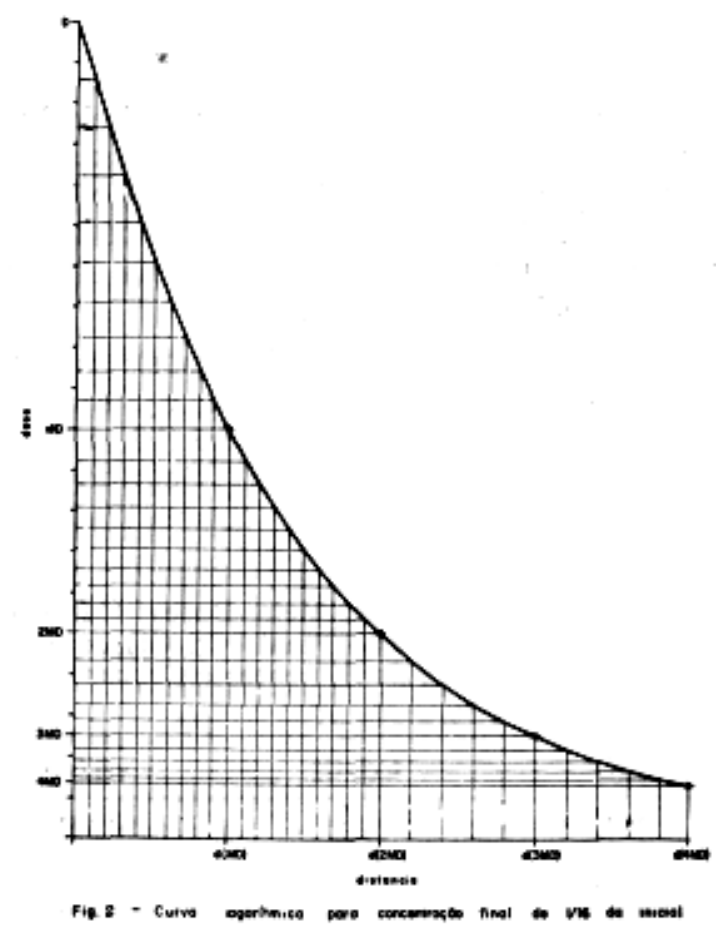

\section{EXEMPLOS DE UTILIZAÇÃO DO PULVERIZADOR-LOGARÍTMICO DETERMINAÇÃO DOS VOLUMES DE DILUENTE E CONCENTRADO}

Admita-se que se pretende construir um pulverizador-logarítmico para ser usado em parcelas de $15 \mathrm{~m}$ de compri mento por $1,5 \mathrm{~m}$ de largura, e que o mesmo vai ser equipado com bicos e usar pressão que, quando deslocado à velocidade de $1 \mathrm{~m} / \mathrm{s}$, tem o débito de 3001 / ha.

A fórmula 2 permite determinar $\mathrm{o}$ volume de diluente a utilizar e, portanto, 


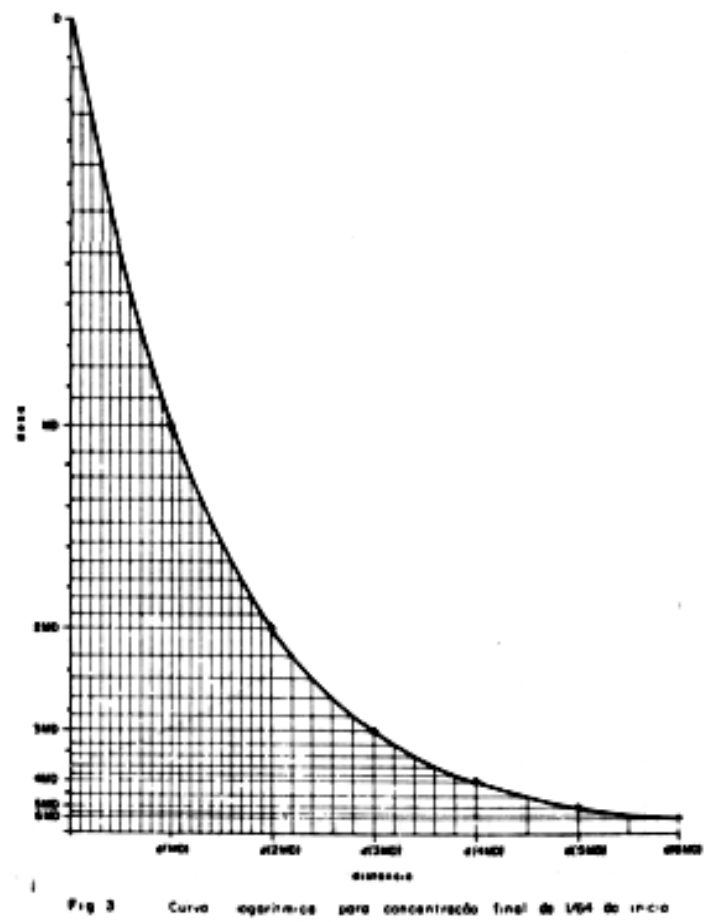

a capacidade mínima do respectivo reservatório :
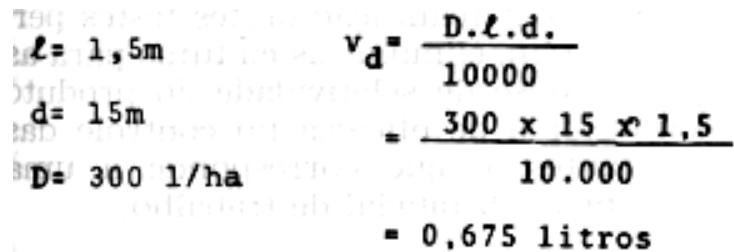

Estabelecendo-se que se deseja atingir, no final da parcela, uma concentração da calda igual a $1 / 16$ da inicial, calcula-se o volume de concentrado a usar a partir da fórmula 3 :

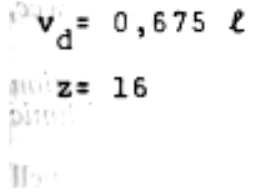

$$
\begin{aligned}
v_{c} & =\frac{0,4343}{\log z} v_{d} \\
& =\frac{0,4343}{\log 16} v_{d} \\
& =0,2431 \text { itros }
\end{aligned}
$$

Pode obter-se este resultado mais facilmente recorrendo ao Quadro 1. Verifica-se que o valor de vd/vc corresponde à concentração de $\mathrm{Cd} / \mathrm{C}_{\mathbf{i}}=1 / 16$, é:

$$
v_{d} / v_{c}=2,773
$$

Sendo vd $=0,675$, vem :

$$
v_{c}=0.2431 \text { itros }
$$

\section{CALCULO DAS DOSES DE APLICAÇÃO}

Suponhamos que o aparelho com estas especificações foi utilizado para testar a seletividade de um produto em diver sas culturas e, também, a eficácia sobre as plantas daninhas presentes no terreno, tendo-se escolhido a concentração inicial de 51 / ha.

Posteriormente, quando da avaliação dos resultados, verificou-se que o produto foi seletivo para uma dada cultura a partir da distância de $3,0 \mathrm{~m}$ e controlou satisfatoriamente as infestantes até aos 10,0m.

Para calcular as doses de aplicação a essas distâncias pode usar-se a fórmula 1 ou recorrer a ábacos.

$\mathrm{Na}$ primeira hipótese determina-se o volume de calda que foi gasto na pulverização da parcela até aos $3 \mathrm{~m}$, e até aos $10 \mathrm{~m}$, recorrendo à fórmula 2 : ,

$$
v_{d}=\frac{\text { D. } . \cdot d .}{10.000}
$$

Para a distância de 3m, vem :

$$
\begin{array}{rlrl}
D & =3001 / \mathrm{ha} & v_{(3 \mathrm{~m})} & =\frac{300 \times 1,5 \times 3}{10.000} \\
\ell & =1,5 \mathrm{~m} & & =0,1351 \mathrm{itros} \\
d=3,0 \mathrm{~m} & & =
\end{array}
$$

Para a distância de $10 \mathrm{~m}$ :

$$
\begin{array}{rlrl}
D & =300 \mathrm{i} / \mathrm{ha} & v_{(10 \mathrm{~m})} & =\frac{300 \times 1,5 \times 10}{10.000} \\
l & =1,5 \mathrm{~m} & & =0,4501 \mathrm{itros} \\
\mathrm{d}=10,0 \mathrm{~m} & &
\end{array}
$$

Substituindo esses valores na fórmula 1 , obtêm-se os valores das doses de aplicação aos $3 \mathrm{~m}$, e aos $10 \mathrm{~m}$. 
Para a distância de $3 \mathrm{~m}$ :

$$
\begin{aligned}
& c_{1}=51 / h a \quad \log c_{(3 n)}=\log 5=0,4343 \frac{0,135}{0,243} \\
& v_{(3 m)}=0,135 l \\
& v_{c}=0,243 t
\end{aligned}
$$

Para a distância de 10m :

$$
\begin{array}{ll}
c_{1}=51 / \text { ha } \quad \log c_{(10 m)} & =10 g 5-0,4343 \frac{0,450}{0,243} \\
v_{(10 m)}=0,450 l & =0,785 \mathrm{l} / \mathrm{ha} \\
v_{c}=0,243 \mathrm{l} &
\end{array}
$$

Conclui-se, pois, que o produto foi seletivo para a cultura até à dose de $2,91 /$ ha e controlou as infestantes a partir dos $0,81 /$ ha.

\section{CONSTRUÇÃO DE ÁBACOS}

Para a construção do ábaco recorrese à fórmula 4 que dá a distância da meiadose do aparelho :

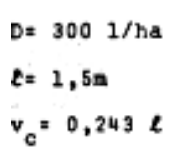

$$
\begin{aligned}
d(M D) & =6931,5 \frac{{ }^{c} c}{D, l_{+}} \\
& =6931,5 \frac{0,243}{300 \times 1,5} \\
& =3,75 n
\end{aligned}
$$

Fica-se, pois, sabendo que a intervalos de $3,75 \mathrm{~m}$ a concentração da calda reduzse, sucessivamente, a metade. Marcando num sistema de coordenadas, as distâncias $3,75,7,5,11,25$, e 15,0m nas abcissas e, nas ordenadas, as doses de 5,0, 2,5, 1,25, 0,625 e 0,312 1/ha, constrói-se o ábaco representado na Figura 4. Por ele pode confirmar-se que à distância de $3 \mathrm{~m}$ a dose de aplicação é de, aproximadamente, 2,9 1 / ha e que à de $10 \mathrm{~m}$, de 0,81 / ha.

Querendo-se recorrer ao ábaco geral da Figura 2, atribui-se a d(MD ), d(2MD ), $\mathrm{d}(3 \mathrm{MD})$ e $\mathrm{d}(4 \mathrm{MD})$, respectivamente, os valores de $3,75,7,5,11,25$ e $15,0 \mathrm{~m}$, e a $\mathrm{D}$, $\mathrm{MD}, 2 \mathrm{MD}, 3 \mathrm{MD}$ e 4MD, as doses de 5,0, $2,5,1,25,0,625$ e 0,312 / ha, obtendo-se os mesmos valores para as doses de aplicação aos 3 e aos $10 \mathrm{~m}$.

Os resultados são, posteriormente,

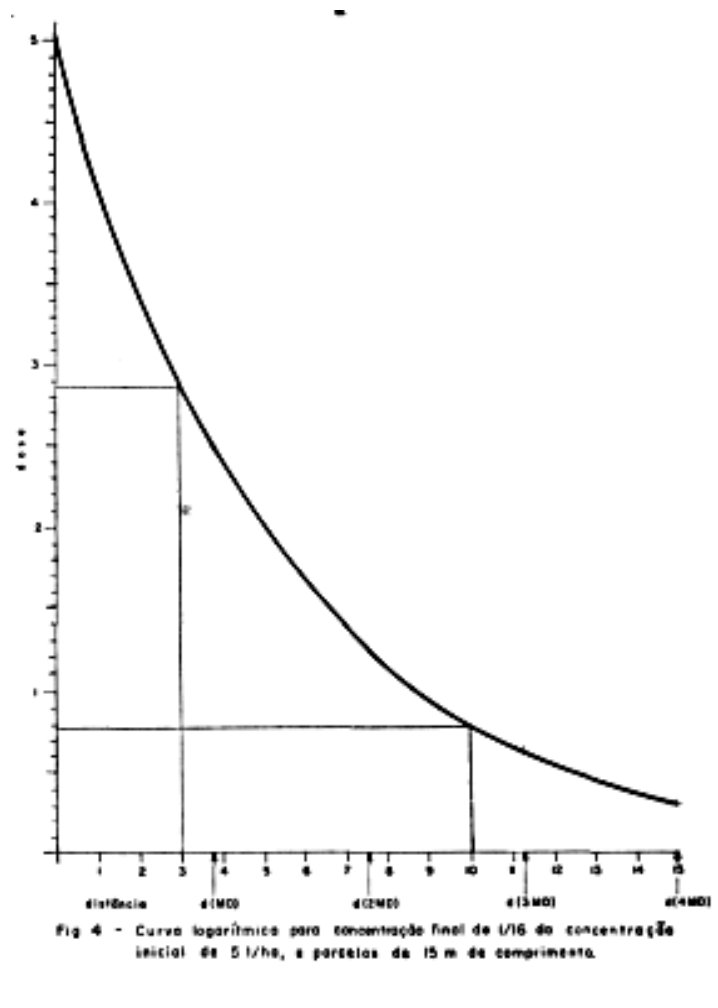

confirmados em ensaios convencionais de campo. A realização destes testes permite, porém, eliminar as culturas para as quais a dose de seletividade do produto é inferior à de eficácia no controle das infestantes, o que corresponde a uma economia substancial de trabalho.

\section{REFERÊNCIAS}

1. Cialone, J.C. \& Bayer, G. - A logarithmic sprayer for small plots. Proc. 16 th Ann. Newcc, 1962.

2. Cialone, J.C.; Bayer, G.; Davis, D.W. - A small plot logarithmic sprayer: some modifications. Proc. 17 th Ann. Newcc, 1963.

3. Cialone, J.C. \& Davis, D.W. - Construction and uses for a hand operated logarithmic sprayer for small plots.

VC-115, Dep. of Vegetable Crops, Cornell Univ., N.Y. U.S.A. 1965.

4. Ries, S.K. \& Terry, C.W. - The design and evaluation of a small-plot sprayer. Weeds 1: 160-174 - jan. 1952 . 